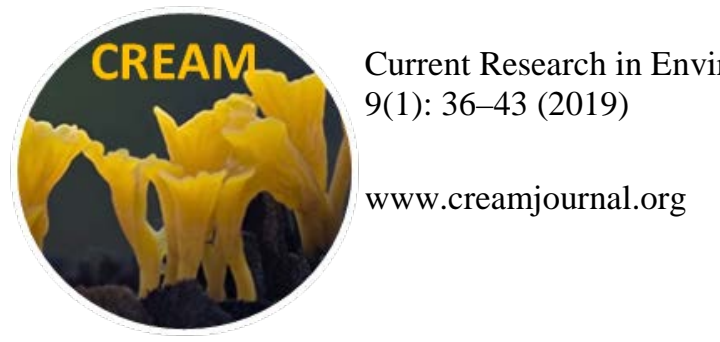

\author{
Article \\ Doi 10.5943/cream/9/1/4
}

\title{
Two new species of Mycena Section Longisetae from Mexico
}

\section{Cortés-Pérez $A^{1}$, Desjardin $D E^{2}$, Perry BA ${ }^{3}$, Ramírez-Cruz $V^{4}$, Ramírez-Guillén $\mathrm{F}^{5}$ and Villalobos-Arámbula $\mathrm{AR}^{6}$}

${ }^{1}$ Coatepec, Veracruz, 91500, Mexico

${ }^{2}$ Deparment of Biology, San Francisco State University, 1600 Holloway Ave., San Francisco, CA 94132 USA

${ }^{3}$ Department of Biological Science, California State University East Bay, 25800 Carlos Bee Blvd., Hayward, CA 94542 USA

${ }^{4}$ CONACyT-Departamento de Botánica y Zoología, Universidad de Guadalajara, 1-139, Zapopan, Jalisco, 45101, Mexico

${ }^{5}$ Instituto de Ecología, A. C., 63, Xalapa, Veracruz, 91000, Mexico

${ }^{6}$ Departamento de Biología Celular y Molecular. Universidad de Guadalajara, 1-139, Zapopan, Jalisco, 41501, Mexico

Cortés-Pérez A, Desjardin DE, Perry BA, Ramírez-Cruz V, Ramírez-Guillén F, Villalobos-Arámbula AR 2019 - Two new species of Mycena Section Longisetae from Mexico. Current Research in Environmental \& Applied Mycology (Journal of Fungal Biology) 9(1), 36-43, Doi 10.5943/cream/9/1/4

\begin{abstract}
Two new species of Mycena belonging to Section Longisetae are described from cloud forests of Veracruz State, Mexico, viz., Mycena glaesisetosa and M. punkissima. Sequences of nuc rDNA ITS1-5.8S-ITS2 were generated for barcoding purposes and for comparisons with similar species. Comprehensive descriptions, line drawings of salient micromorphological features, and photographs of each species are provided.
\end{abstract}

Key words - Agaricales - biodiversity - Mycenaceae - pileosetae - taxonomy - 2 new taxa

\section{Introduction}

Mycena section Longisetae A.H. Sm. ex Maas Geest. was redefined by Desjardin \& Horak (2002) based on a redescription of topotypical material of the type species, Mycena longiseta Höhn. Sixteen species are currently known in this section and they have been reported from America, Europe and Asia (Mass Geesteranus \& de Meijer 1998, Desjardin et al. 2002, Aravindakshan \& Manimohan 2011, 2014). The section includes species with the following features: small basidiomes with pallid pilei less than $10 \mathrm{~mm}$ diam, from which arise conspicuous erect, thick-walled, needleshaped, hyaline or brownish pileosetae; narrowly adnate to free, white, non-marginate lamellae; a filiform, ornamented, pallid stipe that arises from a basal disc; ellipsoid, smooth, amyloid basidiospores; no pleurocystidia; with or without cheilocystidia; pileipellis ranging from a hymeniform layer of acanthocysts to a cutis of spinulose hyphae with acanthocyst terminal cells; dextrinoid tramal tissues; abundant caulocystidia; and clamp connections present or absent. Desjardin et al. (2002) subdivided the section into two provisional stirps: Stirps Longisetae, combining species with a pileipellis on primordia and immature basidiomes composed of a cutis of repent, densely spinulose hyphae with acanthocyst terminal cells through which arise numerous pileosetae; and Stirps Brunneisetosa which includes species with a pileipellis on primordia and immature basidiomes 
consisting of a hymeniform layer of acanthocysts through which arise numerous pileosetae, and at maturity it expands to a subhymeniform layer or a cutis of densely spinulose hyphae with scattered acanthocysts. All members of this section form basidiomes in the uppermost layers of litter, on leaves and twigs of dicotyledonous plants, or on palms (Desjardin et al. 2002, Aravindakshan \& Manimohan 2011). It has been suggested that the center of diversity for sect. Longisetae are the wet tropical forests of Southeast Asia (Desjardin et al. 2002, Aravindakshan \& Manimohan 2011, 2014). Herein we provide data on two new species of Mycena section Longisetae from the cloud forests of Veracruz State, Mexico.

\section{Materials \& Methods}

\section{Morphological studies}

Specimens studied were collected in cloud forests from the state of Veracruz, Mexico. All collections studied are deposited at XAL, with duplicates of selected specimens deposited at SFSU (herbarium abbreviations follow Thiers [continuously updated]). Terminology of macroscopic characters follows Largent (1973) and used the terminology for microstructures according to Vellinga (1988). Free-hand sections were made from basidiomes rehydrated in 70\% ethyl alcohol then mounted in a $5 \%$ solution of $\mathrm{KOH}$, Melzer's reagent or Congo red. Basidiospore statistics include; $x_{m}$, the arithmetic mean of the spore length by spore width $( \pm S D)$ for $n$ spores measured for a given species; Q, the ratio of spore length to spore width, expressed as a range for all spores measured; and $\mathrm{Q}_{\mathrm{m}}$, the mean of all $\mathrm{Q}$ values $( \pm \mathrm{SD})$. Basidiospores, cystidia and other important microscopic structures were drawn using a drawing tube mounted on a Leica DME light microscope (Wetzlar, Germany). All measurements represent the minimum and maximum dimensions observed, and measurements in parentheses represent dimensions that are considered outside the normal range. Photographs of the basidiomes were taken with a Nikon D3200 camera (Tokyo, Japan) with a 18-55 mm lens.

\section{DNA extraction and sequencing}

DNA was extracted from two herbarium specimens following the procedures of Doyle \& Doyle (1987) or Aljanabi \& Martinez (1997). Polymerase Chain Reaction (PCR) was performed to amplify the ITS region following the protocol indicated by Guzmán-Dávalos et al. (2003). The primer pairs ITS1F/ITS4S, ITS1F/ITS4, ITS1/ITS4, and ITS5/ITS4 were used to amplify the entire ITS region. In some cases, the amplification of entire ITS was not successful, thus primers ITS1F/ITS2, ITS1/ITS2, and ITS5/ITS5.8S were used to amplify ITS1, and ITS3/ITS4, ITS5.8SR/ITS4, ITS3/ITS4S, and 5.8SR/ITS4S to amplify ITS2 (Vilgalys \& Hester 1990, White et al. 1990, Gardes \& Bruns 1993). PCR products were cleaned using Illustra GFX columns (GE Healthcare, UK). Purified PCR products were sent to University of Arizona Genetics. Sanger Sequencing was provided by James Shira and the team at University of Arizona Genetics Core (via Science Exchange).

\section{Alignments and comparison of sequences}

Sequences were edited and assembled in Chromas 2.6.5 (Technelysium Pty Ltd, Australia) and Sequencer v.4.9 (Gene Codes Inc., Ann Arbor, MI, USA). Comparison of resulting sequences with existing sequences in GenBank was performed using the BLAST algorithm (Altschul et al. 1990) as implemented in Geneious 9.1.8 (Biomatters Ltd. New Zealand). Both sequences have been uploaded to GenBank (MG926692, MG926683).

\section{Results and Discussion}

\section{Taxonomy}

Mycena glaesisetosa A. Cortés-Pérez, Desjardin \& B. A. Perry, sp. nov. Figs 1a-b, 2 MycoBank number: MB828652; Facesoffungi number: FoF06005 
Etymology - glaesum (L.), amber, referring to the amber color of the pileosetae.

Typus - Mexico, Veracruz, Xalapa, Jardín Botánico Francisco Javier Clavijero, 19³0'45.78" N, 9656'36.35" W, elev. 1345 m, 9 Jun 2016, A. Cortés-Pérez 1504 (holotype XAL, isotype SFSU). GenBank: MG926683.

Diagnosis - Pileus white, surface granulose with amber pileosetae. Lamellae adnate, white. Pileipellis hymeniform with acanthocysts and pileosetae. Cystidia marginal fusoid-ventricose or irregularly clavate, densely spinulose with a smooth and long apical portion. Cheilocystidia absent. Caulocystidia subventricose to lageniform with long necks.

Description - Pileus 1-3 mm diam, initially broadly parabolic to hemispheric, expanding to convex or broadly convex to plano-convex, sometimes centrally depressed; margin striate-sulcate, ciliate; surface moist to dry, granulose to pruinose, white, hispidulous from short, erect, brownish to amber pileosetae that are denser at the disc. Context thin, white. Lamellae adnate, distant (8-15 reaching the stipe) with one series of lamellulae, narrow, white; edge concolorous. Stipe 5-8 $\times 0.2-$ $0.5 \mathrm{~mm}$, central to eccentric, cylindrical with a slightly swollen base, hollow; surface of apex smooth, towards the base minutely pubescent, white; arising from a pruinose, white basal disc. Odor indistinct.

Basidiospores 6-8 × 3-4.5 $\mu \mathrm{m},\left[\mathrm{x}_{\mathrm{m}}=7.19 \pm 0.50 \times 3.85 \pm 0.31 \mu \mathrm{m}, \mathrm{Q}=1.5-2.2, \mathrm{Qm}_{\mathrm{m}}=1.88 \pm\right.$ $0.19, \mathrm{n}=54]$, oblong to subcylindrical, smooth, hyaline, amyloid, thin-walled. Basidia 14-22 × 6.5$9 \mu \mathrm{m}$, clavate to cylindrical, 4-spored, with sterigmata 2-3.5 $\mu \mathrm{m}$ long, hyaline, inamyloid. Pleurocystidia absent. Lamellar edge fertile. Cheilocystidia absent. Subhymenium ramose to subcellular, hyaline, inamyloid, non-gelatinous. Pileipellis initially a hymeniform layer of acanthocysts with some interspersed pileosetae, marginal cystidia, and repent spinulose hyphae; at maturity the pileipellis develops into a subhymeniform layer of acanthocysts; hyphae cylindrical, densely spinulose or smooth, hyaline, inamyloid, non-gelatinized; spinulae 1-1.5 $\mu \mathrm{m}$ long. Acanthocysts 10-23 $\times 7-14 \mu \mathrm{m}$, clavate to subglobose, covered with spinulae, thin-walled, hyaline, inamyloid; spinulae 1-1.5 × 0.5-1 $\mu \mathrm{m}$, cylindrical. Pileosetae scattered, 55-455 × 6-20 $\mu \mathrm{m}$ (at the base) $\times 6-35 \mu \mathrm{m}$ (at the apex), cylindrical to subclavate with a broadly obtuse apex, smooth, thickwalled $(2-12 \mu \mathrm{m})$, amber or pale brown, inamyloid. Marginal cystidia (terminal cells at pileus margin) 43-170 × 6-17 $\mu \mathrm{m}$, fusoid to fusoid-ventricose, densely spinulose over the inflated basal portion, hyaline, inamyloid; apex with 1-2 smooth, acicular projections 15-125 × 2-7 $\mu \mathrm{m}$; spinulae 1-3 $\times 0.5 \mu \mathrm{m}$, cylindrical. Pileus trama interwoven; hyphae 2-29 $\mu \mathrm{m}$ diam, cylindrical to inflated, thin-walled $(<0.5 \mu \mathrm{m})$, hyaline, dextrinoid, non-gelatinous. Lamellar trama regular; hyphae, 1.5-11 $\mu \mathrm{m}$ diam, cylindrical, thin-walled, hyaline, dextrinoid, non-gelatinous. Stipitipellis a cutis of repent smooth, non-gelatinized hyphae. Stipe cortical hyphae 1.5-6 $\mu \mathrm{m}$ diam, cylindrical, thin-walled, hyaline, inamyloid; medullary hyphae 2.5-19 $\mu \mathrm{m}$ diam, cylindrical, thin-walled, hyaline, dextrinoid. Caulocystidia 62-136 × 6-17 $\mu \mathrm{m}$, ventricose to sublageniform with necks up $90 \mu \mathrm{m}$ long, smooth, hyaline, inamyloid. Clamp connections absent.

Habit, habitat and known distribution - Solitary or scattered, growing on leaf litter of Quercus spp., in cloud forests from Veracruz, Mexico.

Additional specimens examined - MEXICO, Veracruz, Xalapa, Jardín Botánico Francisco Javier Clavijero, 19³0'45.78" N, 9656'36.35" W, elev. 1345 m, 4 Feb 2015, A. Cortés-Pérez 1281 (XAL); 17 Mar 2015, A. Cortés-Pérez 1307 (XAL); 10 Jun 2015, A. Cortés-Pérez 1324 (XAL); 22 Apr 2016, A. Cortés-Pérez 1462 (XAL).

Notes - Mycena glaesisetosa forms a hymeniform to subhymeniform pileipellis, indicative of members of strips Brunneisetosa sensu Desjardin et al. (2002). A macroscopically similar species is Mycena brunneisetosa Corner described from Singapore, which also forms orange to brownish orange pileosetae. The latter species differs, however, in forming a pileus with an orangish-white disc, subfree lamellae, a stipe that is white at the apex and orangish at the base, sharply acute pileosetae up to $2000 \mu \mathrm{m}$ long, and aculeate caulocystidia measuring 65-120 × 16-30 $\mu \mathrm{m}$ (at the base) $\times$ 8-14 $\mu \mathrm{m}$ (centrally) (Desjardin \& Horak 2002). Mycena clavulifera (Berk. \& Broome) Sacc., described from Sri Lanka (Berkeley \& Broome 1871) and recently reported from Thailand (Desjardin et al. 2002), forms similar broadly obtuse, pigmented pileosetae, but differs in having a cutis-type 
pileipellis of repent, densely spinulose hyphae, and clavate to ventricose marginal cystidia lacking a narrow elongate apical projection. Mycena lomavritha Manim., described from India, is another similar species with white pileus and brownish pileosetae. The latter forms larger pilei (3-10 mm diam), larger basidiospores (8-10 × 4-6 $\mu \mathrm{m}$ ), abundant fusoid-ventricose cheilocystidia with acicular apex, and aculeate pileosetae with subacute apex (Manimohan \& Leelavathy 1988 [as Mycena indica Manim. \& Leelavathy, nom. illeg., non M. indica Sarwal \& Rawla 1983], Aravindakshan \& Manimohan 2011).

Pairwise comparisons of aligned, overlapping ITS sequences of Mycena glaesisetosa (MG926683) with available sequences exhibiting more than $81 \%$ query coverage show $<84 \%$ similarity with several Mycena adscendens Maas Geest. sequences (KT900142, KT900143), a member of sect. Sacchariferae. Highest similarity (90.1-92.8\%) but with only $42-47 \%$ query coverage was with several undetermined Mycena sp. sequences (KP133214, MH216189, MH216190).
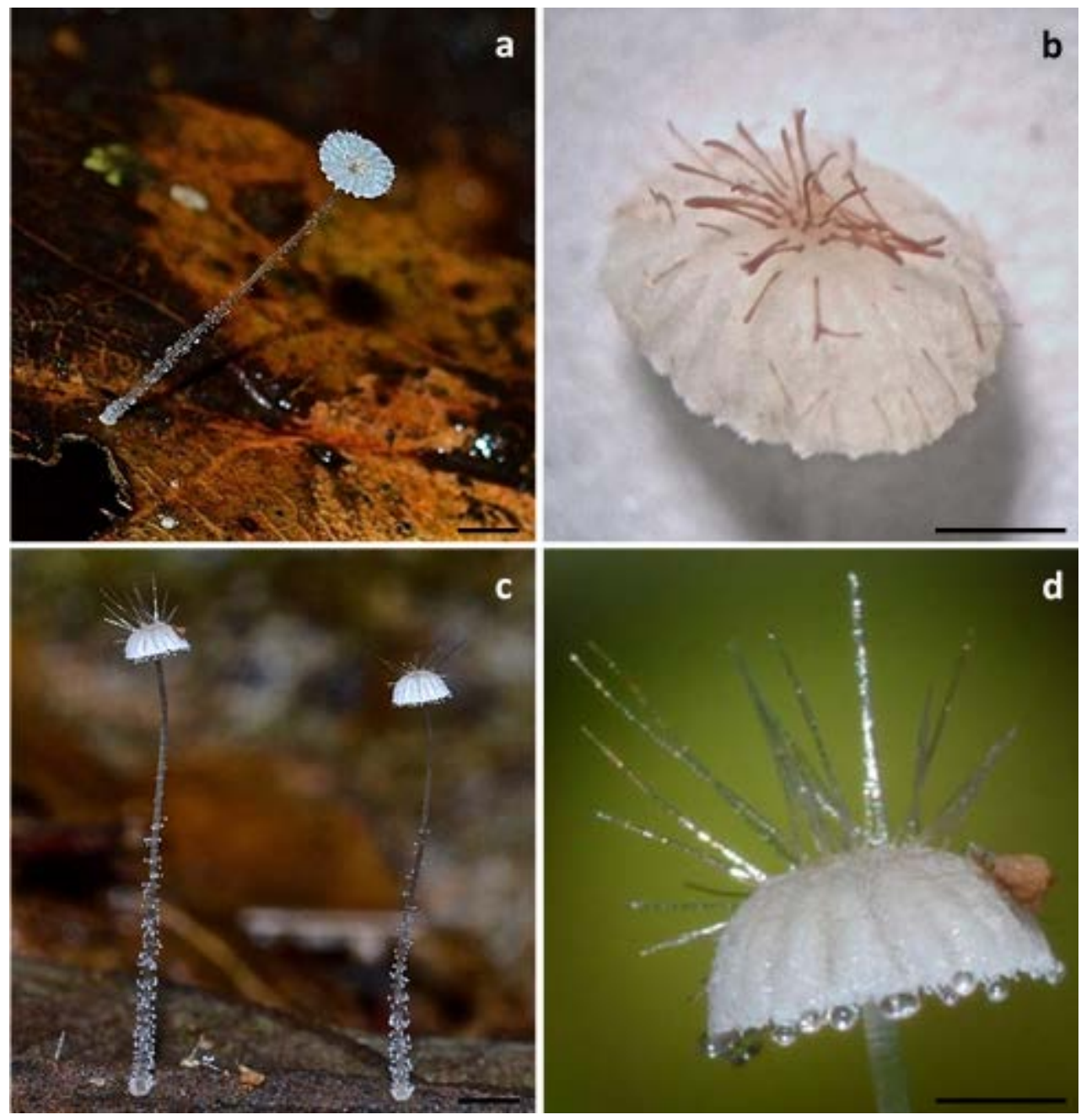

Fig. 1 - a-b Mycena glaesisetosa (A. Cortés-Pérez 1462). c-d Mycena punkissima (A. Cortés-Pérez 1816). Scale bars: a, c $=2 \mathrm{~mm}, \mathrm{~b}, \mathrm{~d}=1 \mathrm{~mm}$.

Mycena punkissima A. Cortés-Pérez, Desjardin \& B. A. Perry, sp. nov. Figs 1c-d, 3

MycoBank number: MB828653; Facesoffungi number: FoF06004

Etymology - named like that because it remembers to the punk counterculture and the punk hairstyle.

Typus - Mexico, Veracruz, Coatepec, Cinco Palos, N19²9’35.37”, W9700’13.31”, elev. 1663 m, 2 Jun 2016; collector A. Cortés-Pérez 1478 (holotype XAL, isotype SFSU). GenBank: MG926692. 
Diagnosis - Pileus white to grayish white, surface granular with pileosetae up $2 \mathrm{~mm}$ long. Pilleipelis a hymeniform layer of acanthocysts with hyaline pileosetae. Marginal cystidia subclavate to cylindrical, densely spinulose with an obtuse and smooth apex, hyaline. Caulocystidia, cylindrical to irregularly cylindrical, hyaline.

Description - Pileus 1-4 mm diam, initially broadly hemispheric to hemispheric-convex, expanding to convex or plano-convex; margin striate, ciliate; surface granular, moist to dry, covered with erect, hyaline pileosetae, up $2 \mathrm{~mm}$ long that are denser at the disc; white or grayish white in the disc and on the striations, white elsewhere. Context thin, white. Lamellae adnexed, distant (8-12 reaching the stipe) with one series of lamellulae, narrow, white; edge concolorous. Stipe 5-16 × $0.2-$ $0.5 \mathrm{~mm}$, central to eccentric, cylindrical with a slightly swollen base, hollow; surface of apex smooth, pubescent towards the middle and the base, white; arising from a pruinose basal disc, white. Odor indistinct.

Basidiospores (6-)7-9(-10) × 3.5-4.5(-5) $\mu \mathrm{m} ;\left[\mathrm{x}_{\mathrm{m}}=7.4 \pm 0.77 \times 4.1 \pm 0.34 \mu \mathrm{m}, \mathrm{Q}=1.5-2.2\right.$, $\mathrm{Q}_{\mathrm{m}}=1.82 \pm 0.16, \mathrm{n}=60$ ], oblong to subcylindrical, smooth, hyaline, amyloid, thin-walled. Basidia $(16-) 17-21(-23) \times 7-8(-9) \mu \mathrm{m}$, clavate to cylindrical, 4-spored, with sterigmata $2-3.5 \mu \mathrm{m}$ long, hyaline, inamyloid. Pleurocystidia absent. Cheilocystidia absent. Subhymenium ramose to subcellular, hyaline, inamyloid, non-gelatinous. Pileipellis initially a hymeniform layer of acanthocysts with some interspersed pileosetae, marginal cystidia, and repent spinulose or smooth hyphae; at maturity the pileipellis develops into a subhymeniform layer of acanthocysts; hyphae cylindrical, densely spinulose or smooth, hyaline, inamyloid, non-gelatinized; spinulae 1-1.5 $\mu \mathrm{m}$ long. Acanthocysts 7-20 × 6-17 $\mu \mathrm{m}$, subclavate to subglobose, covered with spinulae, thick-walled, hyaline, inamyloid, non-gelatinous; spinulae 1-1.5 × 0.5-1 $\mu \mathrm{m}$, cylindrical. Pileosetae 118-1930 $\times$ 11-39 (at the base) $\times 9-20$ (at the apex) $\mu \mathrm{m}$, cylindrical to acicular with a subacute apex, smooth, thick-walled (1-13 $\mu \mathrm{m})$, hyaline, inamyloid. Marginal cystidia (terminal cells at pileus margin) 52$151 \times 8-18 \mu \mathrm{m}$, subclavate to cylindrical, densely spinulose, hyaline, inamyloid; apex obtuse, typically smooth (non-spinulose), seldom spinulose, smooth portion 10-60 × 4-14 $\mu \mathrm{m}$; spinulae 1$3 \times 0.5 \mu \mathrm{m}$, cylindrical. Pileus trama hyphae 3-30 $\mu \mathrm{m}$ diam, cylindrical, thin-walled, hyaline, dextrinoid, non-gelatinous. Lamellar trama hyphae 2-17 $\mu \mathrm{m}$ diam, cylindrical, thin-walled, hyaline, dextrinoid, non-gelatinous. Stipitipellis a cutis of repent smooth, non-gelatinized hyphae. Stipe cortical hyphae 2-11 $\mu \mathrm{m}$ diam, cylindrical, thin-walled, hyaline, inamyloid; medullary hyphae 2-23 $\mu \mathrm{m}$ diam, cylindrical, thin-walled, hyaline, dextrinoid. Caulocystidia 33-137 $\times 12-28 \mu \mathrm{m}$, subcylindrical, smooth, hyaline, inamyloid. Clamp connections absent.

Habit, habitat and known distribution - Solitary or scattered, growing on leaf litter of Clethra mexicana DC. in cloud forests from Veracruz, Mexico.

Additional specimens examined - MEXICO, Veracruz, Coatepec, Cinco Palos, N19²9’35.37”, W9700’13.31”, elev. 1633 m; 10 Jun 2016, A. Cortés-Pérez 1508 (XAL); 17 Jun 2016, A. Cortés-Pérez 1529 (XAL); 17 Aug 2016, A. Cortés-Pérez 1605 (XAL); 15 Oct 2016, A. Cortés-Pérez 1816 (XAL); 5 Jul 2017, A. Cortés-Pérez 1861 (XAL); 15 Oct 2017, A. Cortés-Pérez 1911 (XAL).

Notes - This species belongs to strips Brunneisetosa sensu Desjardin et al. (2002) based on a hymeniform pileipellis in primordia and subhymeniform pileipellis in mature pileus. A macroscopically similar species is Mycena longiseta Höhn., described from Indonesia. The latter species has a white to grey pileus with a ciliate margin and long, erect, hyaline pileosetae, but differs microscopically in having a subgelatinous cutis-type pileipellis, marginal cystidia with attenuated apices, pileosetae only up to $1000 \mu \mathrm{m}$ long, and aculeate caulocystidia with a swollen base (Desjardin \& Horak 2002). Mycena saloma Aravindakshan \& Manim., described from India, is another phenetically similar species which differs in having an initially pure white pileus that discolors with age, by the shorter $(17-82 \times 6.5-20 \mu \mathrm{m})$, clavate to fusiform marginal cystidia that are entirely verrucose or often with a smooth apical prolongation, and by forming shorter pileosetae 60-780 $\times$ 15-30 $\mu \mathrm{m}$ (base) $\times$ 10-11 (apex) $\mu \mathrm{m}$ (Aravindakshan \& Manimohan 2011). Mycena rhaphidocephala Mass G. \& de Meijer, described from Brazil, is macroscopically similar with a pileus covered with pileosetae up $3000 \mu \mathrm{m}$ long. It differs, however, in forming free lamellae, larger 
basidiospores $(9-10.7 \times 4.5-5.4 \mu \mathrm{m})$, shorter marginal cystidia $(45-65 \times 13.5-21.5 \mu \mathrm{m})$ with a slender neck (30-55 × 3.5-5.5 $\mu \mathrm{m}$ ), and by lageniform caulocystidia -75 (or longer) $\times 13.5-18$ (base) $\times$ 5.5-9 (apex) $\mu \mathrm{m}$ (Mass Geesteranus \& de Meijer 1998).

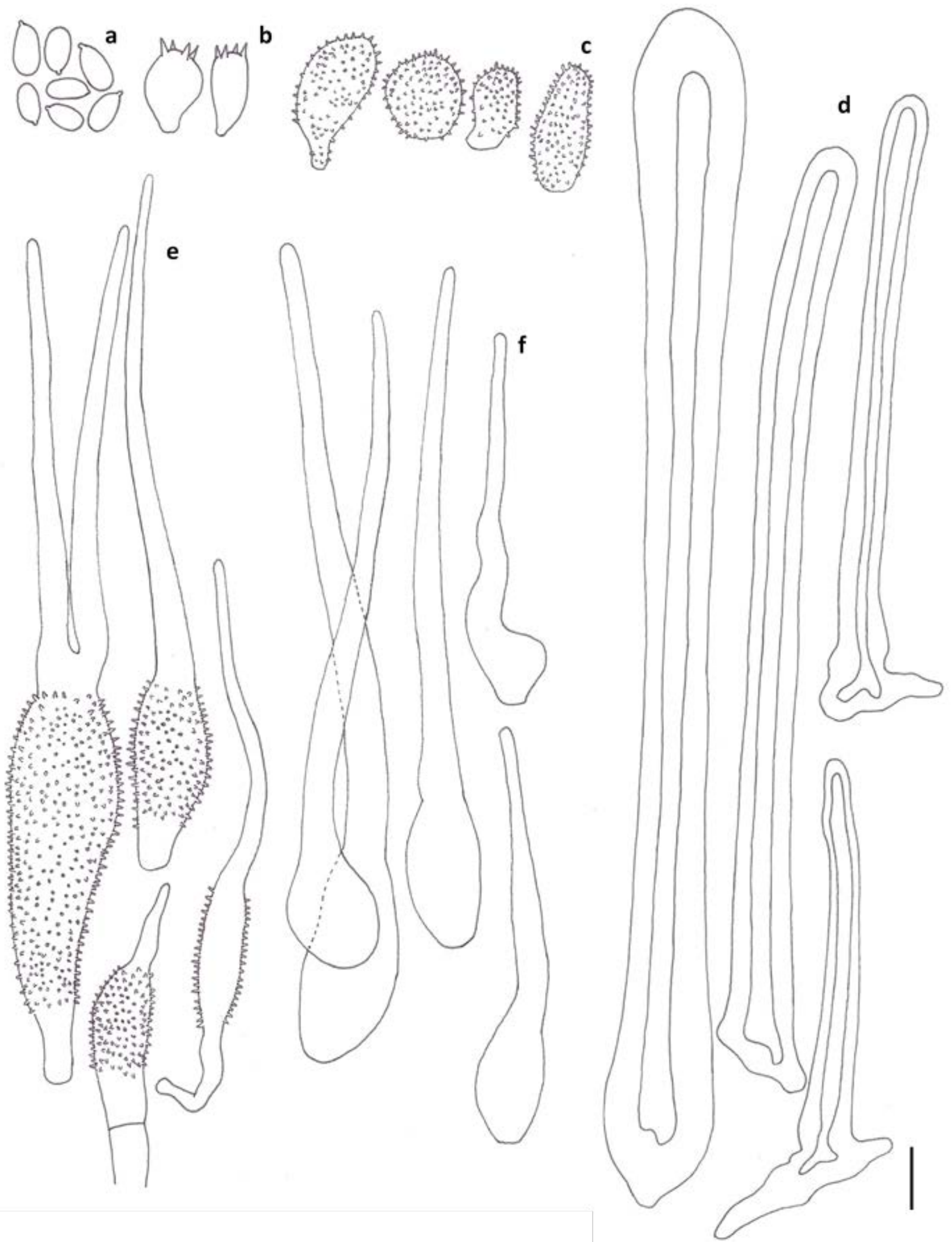

Fig. 2 - Micromorphological features of Mycena glaesisetosa. a Basidiospores. b Basidia. c Acanthocysts. d Pileosetae. e Marginal cystidia. f Caulocystidia. (a-f A. Cortés-Pérez 1324). Scale bars: a-c, e-f $=10 \mu \mathrm{m}$; $d=25 \mu \mathrm{m}$. 


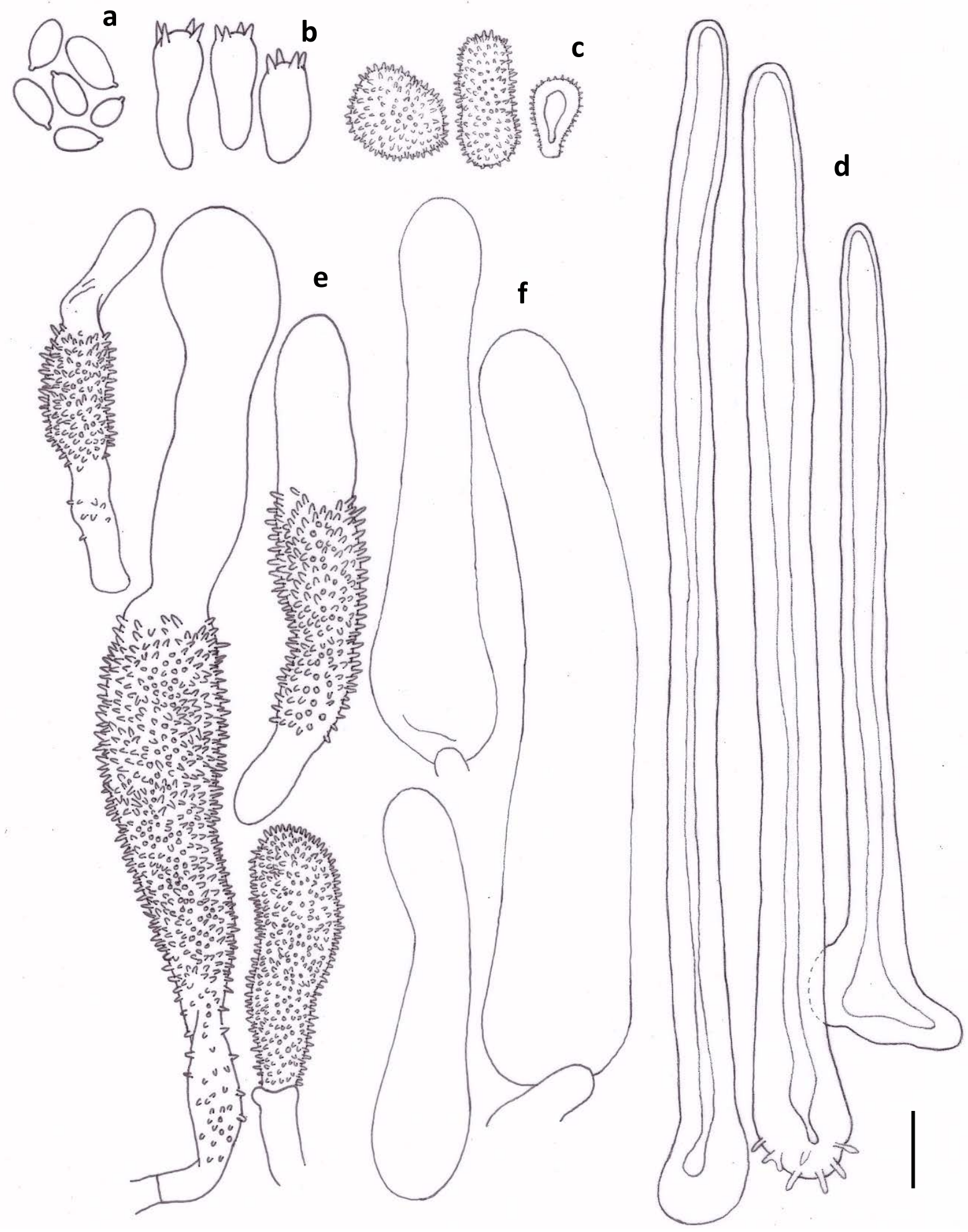

Fig. 3 - Micromorphological features of Mycena punkissima. a Basidiospores. b Basidia. c Acanthocysts. d Pileosetae. e Marginal cystidia. f Caulocystidia. (a-f A. Cortés-Pérez 1478). Scale bars: a-c, e-f $=10 \mu \mathrm{m}$; $d=25 \mu \mathrm{m}$.

Pairwise comparisons of aligned, overlapping ITS sequences of Mycena punkissima (MG926692) with available sequences exhibiting more than 93\% query coverage show <87\% similarity with several Mycena adscendens Maas Geest. sequences (KT900140-KT900143), a 
member of sect. Sacchariferae. Highest similarity (94.5-94.8\%) but with only 52\% query coverage was with several undetermined Mycena sp. sequences (KU861565-KU861567).

\section{Acknowledgements}

The authors thank to the reviewers of this work for their valuable suggestions. We also thank to Manuel Hernández, Cesar Kevin Pérez and Juan Lara Carmona for their help with figures and herbarium assistance at XAL.

\section{References}

Aljanabi SM, Martinez I. 1997 - Universal and rapid salt-extraction of high quality genomic DNA for PCR-based techniques. Nucleic Acids Research. 25:4692-4693.

Altschul SF, Gish W, Miller W, Myers EW, Lipman DJ. 1990 - Basic local alignment search tool. Journal of Molecular Biology 215: 403-410.

Aravindakshan DM, Manimohan P. 2011 - Mycena sect. Longisetae: a new species, a new name, and an addition. Mycotaxon 117: 239-246.

Aravindakshan DM, Manimohan P. 2014 - Three new species of Mycena sect. Longisetae. Mycosphere 5(2): 290-297. Doi 10.5943/mycosphere/5/2/3

Berkeley MJ, Broome CE. 1871 - The fungi of Ceylon (Hymenomycetes, from Agaricus to Cantharellus). Botanical journal of the Linnean Society 11: 494-567.

Desjardin DE, Horak E. 2002 - Agaricales of Indonesia. 4. Mycena sect. Longisetae with comments on allied species. Sydowia 54(2): 142-156.

Desjardin DE, Boonpratuang T, Hywel-Jones NL. 2002- An accounting of the worldwide members of Mycena sect. Longisetae. Fungal Diversity 11: 69-85.

Doyle JJ, Doyle JL. 1987 - A rapid DNA isolation procedure for small quantities of fresh leaf tissue. Phytochemical Bulletin 19:11-15.

Gardes M, Bruns TD. 1993 - ITS primers with enhanced specificity for basidiomycetes - application to the identification of mycorrhizae and rusts. Molecular Ecology 2:113-118, doi:10.1007/s00572-010-0355-X

Guzmán-Dávalos L, Mueller GM, Cifuentes J, Miller AN, Santerre A. 2003 - Traditional infrageneric classification of Gymnopilus is not supported by ribosomal DNA sequence data. Mycologia 95:1204-1214.

Largent DL. 1973 - How to identify mushrooms to genus I: macroscopic features. Mad River Press Inc., Eureka, CA. 86 p.

Mass Geesteranus RA, de Meijer AAR. 1998 - Further Mycenas from the state of Paraná, Brazil. Persoonia 17: 29-46.

Manimohan P, Leelavathy KM. 1988 - Mycena indica, a new species from southern India. Mycologia 80: 861-862.

Sarwal BM, Rawla GS. 1983 - Mycena indica sp. nov. From India. Current Science 52: 564-565.

Thiers B. [continuously updated]. Index Herbariorum: a global directory of public herbaria and associated staff. New York Botanical Graden’s Virtual Herbarium. [cited 2019 Feb 15]. Available from: http://sweetgum.nybg.org/science/ih/

Vellinga EC. 1988 - Glossary. In: Bas, C., Kuyper, Th. W., Noordeloos, M. E. \& Vellinga E. C. (Eds). Flora Agaricina Neerlandica Vol. I. Rotterdam, AA Balkema. pp. 54-64.

Vilgalys R, Hester M. 1990 - Rapid genetic identification and mapping of enzymatically amplified ribosomal DNA from several Cryptococcus species. Journal of Bacteriology 172:4238-4246.

White TJ, Bruns T, Lee S, Taylor J. 1990 - Amplification and direct sequencing of fungal ribosoma RNA genes for phylogenetics. In: Innis MA, Gelfand DH, Sninsky JJ, White TJ, eds. PCR protocols: a guide to methods and applications. San Diego, California: Academic Press. p. 315322. 